\title{
Oral Health Related Quality of Life (OHRQoL) Among Malaysian Children: A Systematic Review
}

Muhammad Zaki Ramli, Nina Suhaity Azmi

Faculty of Industrial Science and Technology, Universiti Malaysia Pahang, Gambang, Kuantan, Malaysia

Presenter: Muhammad Zaki Ramli

Introduction: Oral caries is a major public health issue around the globe, and it is the most prevalent noncommunicable disease (NCD). Children are mostly the population affected by this disease, which affects their quality of life. Therefore, measures to quantify this aspects of quality of life should be established to investigate the current status and to develop future intervention and prevention. The objective of this article is to systematically review the studies related to children's quality of life with regard to their oral health among Malaysian population. Materials and Method: Scopus-indexed articles were retrieved following an advanced search with the following search string, TITLE-ABS-KEY((oral OR dental) AND (child* OR kid* OR (primary dentition)) AND (quality of life) AND Malaysia*), resulting in a final of eight shortlisted and reviewed articles based on the review objective. Results: A few valid and reliable instruments were tested and validated, namely Malaysian version of Early Childhood Oral Health Impact Scale (ECOHIS), Malay Child Oral Impacts on Daily Performances (Malay Child-OIDP), Malaysian version of the Oral Health Impact Profile questionnaire (OHIP-14), and the most commonly used child oral-health-related quality-of-life (OHRQoL). However, Dental Aesthetic Index (DAl) score did not predict the effect of malocclusion on the OHRQoL. Oral caries was highly prevalent among Malaysian Orang Asli children. Oral health did not only have an impact on quality of life in Malaysian preschool children, but also on their caregivers. A health-promoting school model namely the Doktor Muda Programme for primary schools in Malaysia showed some positive impacts on children's oral health related quality of life. Conclusion: Further research among Malaysian children population with regard to their oral health related quality of life should be continued for the benefits of communities at large. 